\title{
Comparación de efectividad de las técnicas de educción de requisitos software: visión novel y experta
}

\author{
Comparison on effectiveness of the software requirements elicitation techniques: \\ novice and expert vision \\ Dante Carrizo Moreno ${ }^{1}$
}

Recibido 27 de marzo de 2012, aceptado 24 de agosto de 2012

Received: March 27, 2012 Accepted: August 24, 2012

\begin{abstract}
RESUMEN
La Ingeniería de Requisitos puede hacer uso de una gran cantidad de técnicas para educir las necesidades de los usuarios. No obstante, apenas existen guías y criterios prácticos para realizar la selección de técnicas en un proyecto de desarrollo de software. Este artículo intenta conocer la visión que tienen los ingenieros de requisitos noveles acerca de la efectividad de las técnicas de educción y compararlas con la visión de expertos en requisitos. Para efectuar la comparación se utiliza la técnica de emparrillado que permite conocer indirectamente la opinión de los sujetos respecto de las técnicas. Los resultados muestran una sustancial diferencia entre ambas visiones respecto a la efectividad de las técnicas y contexto de la educción. Esto implica que una más amplia formación, y principalmente práctica, es necesaria para que los ingenieros noveles puedan reconocer diferencias de efectividad de las técnicas y poder decidir con mayor certeza sobre las técnicas más adecuadas a utilizar en las sesiones de educción de requisitos.

Palabras clave: Ingeniería de requisitos, educción de requisitos, técnicas de educción, atributos contextuales, emparrillado.
\end{abstract}

\begin{abstract}
Requirements engineering can use a lot of techniques to gather the users' needs. However, currently there are few practical guidelines and criteria for selecting techniques in a software development project. This paper tries to know the vision that novice requirements engineers have about the effectiveness of requirements elicitation techniques and compare it with the requirements experts'vision. To carry out the comparison, repertory grid technique was used. This technique allows knowing indirectly the subject's opinion on the techniques. The results show a substantial difference between both visions with regard to the techniques and elicitation context. This implies that a more extensive training, and primarily practice, is necessary in order for novice engineers to recognize differences in techniques effectiveness and decide with greater certainty about the most appropriate techniques to use in requirements elicitation sessions.
\end{abstract}

Keywords: Requirements engineering, requirements elicitation, elicitation techniques, contextual attributes, repertory grid.

\section{INTRODUCCIÓN}

La actividad de determinar los requisitos que debe cumplir o satisfacer el sistema software final se ha denominado en la literatura de diversas formas, aunque en los últimos años se ha popularizado el término de Ingeniería de Requisitos (IR). De igual forma hay multitud de visiones del proceso

1 Departamento de Informática y Ciencias de la Computación. Universidad de Atacama. Avda. Copayapu 485. Copiapó, Chile. E-mail: dante.carrizo@uda.cl 
de IR, pero actualmente se ha consensuado un conjunto de actividades a llevar a cabo: Educción, Análisis, Especificación, Validación y Gestión de Requisitos [1].

La educción de requisitos comprende, principalmente, la captura y descubrimiento de las necesidades de los usuarios/clientes. Su objetivo es identificar información que determine las características deseadas del sistema software. Para esto, también es necesario considerar información acerca del dominio del problema así como la que poseen las personas que tienen algún interés en el desarrollo del producto (stakeholders). El conocimiento del dominio del problema ayuda al analista a obtener un grado común de entendimiento del mundo del usuario y facilita la captura y especificación de requisitos.

En la práctica, cuando un analista o ingeniero de requisitos se enfrenta a la tarea de determinar los requisitos de un sistema software, muy a menudo utiliza únicamente las entrevistas para la captura de información. A pesar, incluso, de que conoce varias otras técnicas [2]. Esto puede deberse al desconocimiento de las ventajas de cada técnica, a la falta de una metodología que le guíe en este proceso, o a la forma ya arraigada de trabajar: en muchos casos, un método o técnica de educción es elegido no por sus características o ventajas, sino simplemente por razones de historia o familiaridad [3].

Algunas revisiones dan cuenta de las decenas de técnicas de educción que existen [4-7]. Estos estudios muestran que las técnicas de educción son por naturaleza muy diferentes. De esta forma, se puede esperar que algunas de ellas puedan desempeñarse mejor en unas situaciones que en otras. Pero, pese a esta convicción, la mayoría de los ingenieros de desarrollo de software desconoce esta diversidad de técnicas perdiéndose la oportunidad de optimizar la educción de requisitos. Esta situación refleja un ejemplo más de la ya conocida brecha entre la teoría y la práctica [8].

Este trabajo pretende conocer la visión consensuada que tienen los ingenieros de requisitos o analistas noveles acerca de la efectividad de las técnicas de educción de requisitos y compararla con la visión de expertos en el área. Para obtener estas visiones se utiliza una técnica proveniente de la psicología, el emparrillado (repertory grid). Esta técnica establece similitudes y diferencias entre un conjunto de elementos y constructos o características de un dominio específico. En este caso, los elementos corresponden a las técnicas de educción de requisitos seleccionadas para el estudio y las características corresponden a los atributos del contexto, especialmente aquellos relacionados con los participantes en el proceso, que influyen en la efectividad de estas técnicas.

La comparación de estas visiones puede ayudar: a los investigadores, a mejorar el diseño de futuros estudios empíricos; y a los académicos, a la mejora de estrategias para enseñar las técnicas de educción de requisitos. Los resultados de estas mejoras ayudarían a los desarrolladores a realizar un proceso de educción de requisitos óptimo, produciendo un documento de especificación de requisitos correcto $\mathrm{y}$, por lo tanto, un producto software final de calidad.

El resto del documento se estructura como sigue: en la segunda sección se resume la técnica de emparrillado utilizada en el estudio. En la tercera sección se presenta una discusión sobre los trabajos previos relacionados con el problema. La cuarta sección expone la metodología utilizada para llevar a cabo el estudio. En la quinta sección se presenta el diseño de la parrilla, estableciendo los elementos y las características considerados en el trabajo. Posteriormente, en la sexta sección se presenta el desarrollo del estudio, tanto para los ingenieros noveles como para los expertos. En la sección séptima se presenta la discusión de los resultados del estudio, comparando ambas visiones. Finalmente, en la última sección se exponen las conclusiones finales y trabajo futuro.

\section{BACKGROUND}

La técnica de emparrillado (repertory grid) fue presentada por George Kelly en el 1955 [9]. Esta técnica se basa en la teoría de los constructos personales, originada en la psicología, que establece que las personas interpretan el mundo en términos de su propio conjunto de abstracciones bipolares que usan para distinguir entre elementos similares y diferentes. La técnica del emparrillado es utilizada, inicialmente, en la adquisición de conocimientos para la construcción de sistemas basados en conocimientos [10] y, en los últimos años, en la 
captura de requisitos para el desarrollo de productos software [11].

Un emparrillado es, esencialmente, un test complejo de clasificación en el cual se establece una lista de elementos en base a un conjunto bipolar de características. Cada característica se define como una dimensión de escala bipolar interna que extrae la similitud de un conjunto de elementos y la diferencia de este conjunto de elementos de los otros.

En la práctica, los valores otorgados por el sujeto de estudio se introducen en una tabla bidimensional, como la de la Tabla 1, en la cual hay una columna por cada elemento $\mathrm{E}$ y una fila por cada constructo o característica $\mathrm{C}$. Cada intersección fila-columna en la tabla contiene un valor $\mathrm{v}$ indicando cómo una característica dada se considera para un elemento particular. Generalmente, hay tres estrategias de diseñar la parrilla: dicotómica, es decir, los valores asignados son 0 o 1 dependiendo de si existe o no la característica para cada elemento; clasificatoria, donde se asigna para cada uno de los $n$ elementos un valor entre 1 y n dependiendo del orden lógico que tenga esa característica; y evaluativa asignando un valor entre 1 y k valorando la característica entre dos polos opuestos.

Tabla 1. Parrilla de valores.

\begin{tabular}{|c|c|c|c|c|c|}
\cline { 2 - 6 } \multicolumn{1}{c|}{} & $\mathbf{E}_{\mathbf{1}}$ & $\mathbf{E}_{\mathbf{2}}$ & $\mathbf{E}_{\mathbf{3}}$ & $\ldots$ & $\mathbf{E}_{\mathbf{n}}$ \\
\hline $\mathbf{C}_{\mathbf{1}}$ & $v$ & $v$ & $v$ & $v$ & $v$ \\
\hline $\mathbf{C}_{\mathbf{2}}$ & $v$ & $v$ & $v$ & $v$ & $v$ \\
\hline $\mathbf{C}_{\mathbf{3}}$ & $v$ & $v$ & $v$ & $v$ & $v$ \\
\hline$\ldots$ & $v$ & $v$ & $v$ & $v$ & $v$ \\
\hline $\mathbf{C}_{\mathbf{m}}$ & $v$ & $v$ & $v$ & $v$ & $v$ \\
\hline
\end{tabular}

Para el presente estudio, los elementos serán las técnicas de educción de requisitos seleccionadas, desde un conjunto finito, como las más populares. Estas técnicas deben ser conocidas por todos los sujetos para que puedan evaluarlas sin problemas. Una técnica desconocida puede sesgar la valorización de la parrilla. Los constructos o características serán los atributos que influyen en la efectividad de las técnicas de educción. Estos atributos son seleccionados de un conjunto de atributos relevantes y tienen que ver con las características de los participantes en el proceso de educción de requisitos, es decir, el ingeniero de requisitos o analista y los poseedores de la información relevantes para conformar los requisitos del producto software. Se utilizará en el estudio una valoración evaluativa entre 1 y 5 ya que los constructos no son dicotómicos, es decir, aceptan valores intermedios, y, además, pueden coincidir en la misma valoración más de una técnica.

Esta parrilla es tratada mediante el cálculo sucesivo de matrices que van uniendo los elementos menos distanciados en su valoración. De la misma forma son tratadas las características. Este análisis de conglomerados (clusters) arroja una gráfica de la similitud de grupos de elementos y características. Además, el método arroja gráficas y datos análisis de componentes principales y medidas de equiparación, entre otros resultados. Este método puede realizarse manualmente, pero en este estudio se utilizará una herramienta computacional denominada Webgrid 5 [12].

\section{TRABAJOS RELACIONADOS}

Junto con las revisiones de las técnicas de educción existen otros estudios que tratan específicamente las comparaciones de efectividad de técnicas. Por un lado están los estudios empíricos, principalmente basados en experimentos, que comparan la efectividad de dos o más técnicas bajo ciertas condiciones particulares del proceso de educción. Estos trabajos están bien analizados por Dieste y otros [13]. Sin embargo, el alcance de los resultados es limitado y no representan visión alguna.

Por otro lado, existen varias propuestas de índole teórica que intentan facilitar la selección de las técnicas de educción mediante una comparación de su efectividad bajo ciertas situaciones contextuales. Un estudio detallado sobre estos trabajos es presentado por Carrizo y otros [14]. Nuevamente, son estudios que representan la visión de autores o de los resultados de otros estudios que compilan. La Tabla 2 muestra en detalle estos trabajos.

Respecto al uso de emparrillado, existen algunos trabajos que han utilizado la técnica para comparar las características de los integrantes de equipos de desarrollos en XP [15] y para comparar las destrezas deseadas para los ingenieros de software [16]. Sin embargo, no hay trabajos que la usen para comparar técnicas del proceso de requisitos. 
Tabla 2. Resumen de trabajos sobre educción.

\begin{tabular}{|c|c|c|c|c|c|c|c|c|c|c|c|}
\hline & 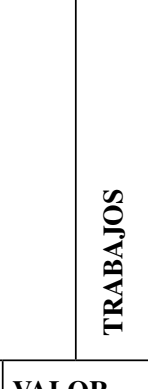 & 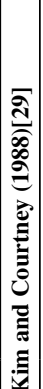 & 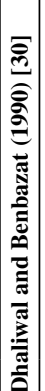 & 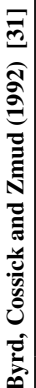 & 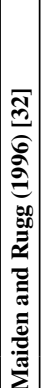 & 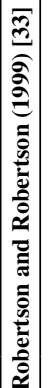 & 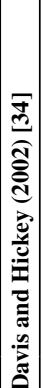 & 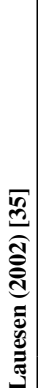 & 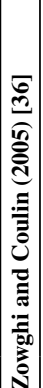 & 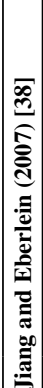 & 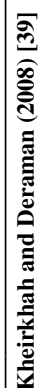 \\
\hline ASPECTO & VALOR & & & - & & & & 2 & & & \\
\hline Area & $\begin{array}{l}\text { Ingeniería de } \\
\text { Software }\end{array}$ & & & $\mathrm{x}$ & $\mathrm{x}$ & $\mathrm{x}$ & $\mathrm{x}$ & $\mathrm{x}$ & $\mathrm{x}$ & $\mathrm{x}$ & $\mathrm{x}$ \\
\hline & $\begin{array}{l}\text { Ingeniería del } \\
\text { Conocimiento }\end{array}$ & $\mathrm{x}$ & $\mathrm{x}$ & & & & & & & & \\
\hline $\begin{array}{l}\text { Proceso de } \\
\text { Requisitos }\end{array}$ & $\begin{array}{l}\text { Proceso } \\
\text { Global }\end{array}$ & & & & & & & & & $\mathrm{x}$ & $\mathrm{x}$ \\
\hline & $\begin{array}{l}\text { Proceso de } \\
\text { Educción }\end{array}$ & $\mathrm{x}$ & $\mathrm{x}$ & $\mathrm{x}$ & $\mathrm{x}$ & $\mathrm{x}$ & $\mathrm{x}$ & $\mathrm{x}$ & $\mathrm{x}$ & & \\
\hline Cobertura de & Bajo (0-5) & $\mathrm{x}$ & & & & & & & & & $\mathrm{x}$ \\
\hline & Medio (6-14) & & & $\mathrm{x}$ & $\mathrm{x}$ & & & & $\mathrm{x}$ & $\mathrm{x}$ & \\
\hline & Alto $(>=15)$ & & $\mathrm{x}$ & & & $\mathrm{x}$ & $\mathrm{x}$ & $\mathrm{x}$ & & & \\
\hline $\begin{array}{l}\text { Factores } \\
\text { Contextuales }\end{array}$ & $\begin{array}{l}\text { Analista/ } \\
\text { Desarrollador }\end{array}$ & & $\mathrm{x}$ & & $\mathrm{x}$ & & $\mathrm{x}$ & & & & \\
\hline & Informante & & $x$ & & $\mathrm{x}$ & & $\mathrm{x}$ & $\mathrm{x}$ & & & \\
\hline & $\begin{array}{l}\text { Dominio del } \\
\text { Problema }\end{array}$ & $\mathrm{x}$ & $\mathrm{x}$ & $\mathrm{x}$ & $\mathrm{x}$ & $\mathrm{x}$ & $\mathrm{x}$ & $\mathrm{x}$ & & & $\mathrm{x}$ \\
\hline & $\begin{array}{l}\text { Dominio de } \\
\text { la Solución }\end{array}$ & & $\mathrm{x}$ & & & & $\mathrm{x}$ & & & & $\mathrm{x}$ \\
\hline & Proceso & & $\mathrm{x}$ & $\mathrm{x}$ & $\mathrm{x}$ & & $\mathrm{x}$ & & $\mathrm{x}$ & $x$ & $\mathrm{x}$ \\
\hline Valoración de & Disponible & & & & & $\mathrm{x}$ & & $\mathrm{x}$ & $\mathrm{x}$ & & \\
\hline & Poco Clara & $\mathrm{x}$ & $\mathrm{x}$ & $\mathrm{x}$ & $\mathrm{x}$ & & $\mathrm{x}$ & & & $\mathrm{x}$ & $\mathrm{x}$ \\
\hline Actualización & Fácil & $\mathrm{x}$ & & $\mathrm{x}$ & $\mathrm{x}$ & & $\mathrm{x}$ & $\mathrm{x}$ & $\mathrm{x}$ & & \\
\hline & Difícil & & $\mathrm{x}$ & & & $\mathrm{x}$ & & & & $x$ & $\mathrm{x}$ \\
\hline $\begin{array}{l}\text { Concepto de } \\
\text { adecuación }\end{array}$ & $\begin{array}{l}\text { Calidad del } \\
\text { Producto }\end{array}$ & & $\mathrm{x}$ & & & & & & & & \\
\hline & Pertinencia & $\mathrm{x}$ & & $\mathrm{x}$ & $\mathrm{x}$ & $\mathrm{x}$ & $\mathrm{x}$ & & $\mathrm{x}$ & $\mathrm{x}$ & \\
\hline & Efectividad & & $\mathrm{x}$ & & & & & $\mathrm{x}$ & & & \\
\hline
\end{tabular}

En resumen, no hay estudios que representen la visión particular de expertos o de ingenieros de requisitos noveles sobre la efectividad de las técnicas.

\section{METODOLOGÍA DE INVESTIGACIÓN}

Para obtener y luego comparar las visiones de los ingenieros de requisitos noveles y de los expertos se procesan las parrillas de cada grupo de sujetos de estudio por separado, y luego se comparan los resultados obtenidos. La metodología aplicada se resume en la Figura 1.

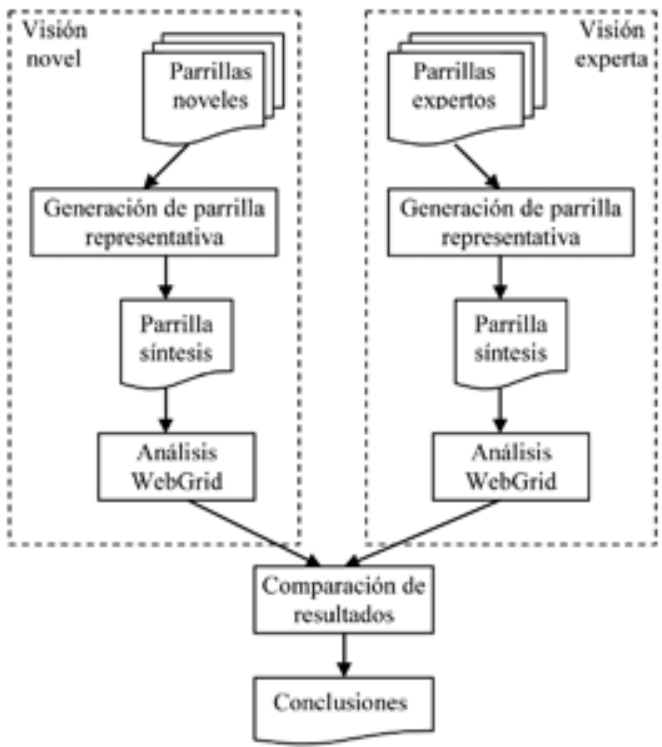

Figura 1. Metodología de investigación.

Para el caso de los noveles se utiliza la valoración hecha por 17 estudiantes de un master en ingeniería de software. Ellos son ingenieros informáticos con poca y similar experiencia en requisitos (entre 2 a 3 años) y que han sido instruidos sobre las técnicas de educción de requisitos. A partir de estas 17 parrillas se genera una parrilla representativa del conjunto con la moda de los valores respectivos. La moda es más pertinente que la media ya que esta última puede entregar un valor que no ha sido considerado por ningún sujeto.

Por otro lado, tres expertos en el área de requisitos valoran la parrilla basados en su experiencia (entre 15 y 20 años) y conocimiento. De igual forma que en el caso anterior, se obtiene una parrilla representativa conformada con las modas de los valores otorgados por los expertos.

La definición de la valoración de las parrillas es una adaptación de la original. La técnica prescribe valores de las características para los elementos. En esta investigación, los constructos no son características de los elementos, sino que son aspectos contextuales que pueden variar el comportamiento de los elementos. Esta modificación permitirá interpretar correctamente los resultados del estudio. 
Finalmente, ambas parrillas son procesadas por medio del software libre disponible en Internet Webgrid 5 obteniendo los resultados gráficos que luego son discutidos y comparados.

\section{DISEÑO DE LA PARRILLA}

\section{Elementos de la parrilla}

La técnica de emparrillado requiere de la identificación de elementos en el dominio que interesa valorar. En este estudio los elementos de la parrilla son las técnicas de educción de requisitos. Es decir, las técnicas que permiten capturar la información necesaria para especificar los requisitos software. Como se indicó arriba, existen decenas de técnicas de educción. Muchas de estas técnicas provienen de áreas como psicología cognitiva, antropología, sociología y lingüística [7], y han sido utilizadas con éxito en Ingeniería del Conocimiento y, últimamente, en Ingeniería de Software.

De este universo de técnicas de educción se han seleccionado para el estudio aquellas que pueden resultar más conocidas para los ingenieros de requisitos. Las técnicas elegidas son descritas y referenciadas en la Tabla 3.

\section{Constructos de la parrilla}

El emparrillado requiere, también, de la identificación de constructos que permitirán caracterizar los elementos de la parrilla mediante la valoración solicitada para cada par elemento/constructo. Los constructos en este estudio están relacionados con los atributos contextuales del proceso de educción de requisitos que influyen en la efectividad de las técnicas de educción. Es decir, en los atributos que al variar producen diferencias en los resultados de la aplicación de una técnica en una sesión de educción.

Los atributos que influyen en la efectividad de las técnicas tienen que ver con diferentes aspectos relacionados con el ingeniero de requisitos, los stakeholders, el dominio de aplicación, el dominio de solución y el proceso mismo [29].

Para este estudio se han considerado los atributos que tienen que ver con los participantes del proceso, es decir: los del ingeniero de requisitos o analista, denominado eductor en el estudio, ya que es la persona que realiza la educción de los requisitos; y
Tabla 3. Técnicas de educción de requisitos consideradas en el estudio.

\begin{tabular}{|c|c|}
\hline Técnica & Descripción \\
\hline $\begin{array}{l}\text { Entrevista no } \\
\text { estructurada } \\
{[17]}\end{array}$ & $\begin{array}{l}\text { Interacción presencial entre un informante } \\
\text { y el eductor en la que no existe estructura } \\
\text { ni definición acabada de objetivos y } \\
\text { contenidos }\end{array}$ \\
\hline $\begin{array}{l}\text { Entrevista } \\
\text { estructurada } \\
{[18]}\end{array}$ & $\begin{array}{l}\text { Interacción presencial entre un informante } \\
\text { y el eductor en la que existe una } \\
\text { preparación de los objetivos y contenidos } \\
\text { de la sesión }\end{array}$ \\
\hline \begin{tabular}{|l|} 
Observación de \\
tareas habituales \\
{$[19]$}
\end{tabular} & $\begin{array}{l}\text { Observación hecha por el eductor de las } \\
\text { personas en su entorno de trabajo }\end{array}$ \\
\hline $\begin{array}{l}\text { Clasificación de } \\
\text { conceptos [20] }\end{array}$ & $\begin{array}{l}\text { El eductor presenta al o los informantes un } \\
\text { conjunto de conceptos del dominio escritos } \\
\text { en cartas pidiéndole diversas acciones }\end{array}$ \\
\hline $\begin{array}{l}\text { Cuestionarios } \\
{[21]}\end{array}$ & $\begin{array}{l}\text { Conjunto de cuestiones en papel u otro } \\
\text { medio presentados o enviados a uno o } \\
\text { varios informantes }\end{array}$ \\
\hline $\begin{array}{l}\text { Anál } \\
\text { proto }\end{array}$ & $\begin{array}{l}\text { El informante cuenta en voz alta lo } \\
\text { que hace cuando se enfrenta a tareas } \\
\text { específicas }\end{array}$ \\
\hline $\begin{array}{l}\text { Emparrillado } \\
{[23]}\end{array}$ & $\begin{array}{l}\text { El informante debe evaluar un conjunto } \\
\text { de elementos del dominio sobre la base } \\
\text { de constructos (características de los } \\
\text { elementos) }\end{array}$ \\
\hline $\begin{array}{l}\text { Torm } \\
\text { Ideas }\end{array}$ & $\begin{array}{l}\text { Generación de ideas, deliberadamente y sin } \\
\text { orden preestablecido, en modo individual o } \\
\text { en grupo, evitando evaluaciones inmediatas }\end{array}$ \\
\hline $\begin{array}{l}\text { Método Delphi } \\
\text { [25] }\end{array}$ & $\begin{array}{l}\text { Retroalimentación de respuestas a un } \\
\text { formulario con el objetivo de obtener un } \\
\text { resultado representativo de la opinión del } \\
\text { grupo }\end{array}$ \\
\hline $\begin{array}{l}\text { Focus group } \\
{[26]}\end{array}$ & $\begin{array}{l}\text { Entrevista grupal semiestructurada donde } \\
\text { se anima a la discusión abierta en presencia } \\
\text { del eductor }\end{array}$ \\
\hline Laddering [27] & $\begin{array}{l}\text { El informante es pedido que a partir de un } \\
\text { concepto semilla del dominio establezca } \\
\text { otros relacionados jerárquicamente con él }\end{array}$ \\
\hline \begin{tabular}{|l} 
Casos de uso \\
{$[28]$}
\end{tabular} & $\begin{array}{l}\text { El informante describe un conjunto } \\
\text { de posibles escenarios de acciones y } \\
\text { eventos que describen una parte del } \\
\text { comportamiento del sistema }\end{array}$ \\
\hline
\end{tabular}

los de los stakeholders, denominados informantes en el estudio, ya que son los que poseen la información relevante para conformar los requisitos del producto. La selección de estos factores se debe a que se quiere considerar un número reducido de atributos para poder realizar un análisis consistente de los resultados considerando solo el factor humano del proceso. Estos atributos contextuales son descritos en la Tabla 4. 
Tabla 4. Atributos contextuales de los participantes considerados en el estudio.

\begin{tabular}{|c|c|c|}
\hline Factor & Atributos & Descripción \\
\hline \multirow[t]{2}{*}{ Eductor } & $\begin{array}{l}\text { Experiencia en } \\
\text { educción }\end{array}$ & $\begin{array}{l}\text { Cantidad de proyectos previos } \\
\text { en que el eductor ha realizado } \\
\text { actividades de educción }\end{array}$ \\
\hline & $\begin{array}{l}\text { Familiaridad } \\
\text { con el dominio }\end{array}$ & $\begin{array}{l}\text { Cantidad de proyectos previos } \\
\text { en el dominio o conocimiento } \\
\text { adquirido en él, por parte del } \\
\text { eductor }\end{array}$ \\
\hline \multirow[t]{5}{*}{ Informante } & $\begin{array}{l}\text { Disponibilidad } \\
\text { de tiempo }\end{array}$ & $\begin{array}{l}\text { Tiempo del que dispone para } \\
\text { dedicar a las sesiones }\end{array}$ \\
\hline & $\begin{array}{l}\text { Interés del } \\
\text { informante }\end{array}$ & $\begin{array}{l}\text { Motivación mostrada por el } \\
\text { informante para participar en } \\
\text { las sesiones de educción }\end{array}$ \\
\hline & $\begin{array}{l}\text { Nivel de } \\
\text { pericia }\end{array}$ & $\begin{array}{l}\text { Experiencia del informante } \\
\text { en el dominio del problema o } \\
\text { su trabajo }\end{array}$ \\
\hline & $\begin{array}{l}\text { Consenso entre } \\
\text { informantes }\end{array}$ & $\begin{array}{l}\text { Grado de acuerdo inicial entre } \\
\text { los informantes }\end{array}$ \\
\hline & $\begin{array}{l}\text { Capacidad de } \\
\text { articulación }\end{array}$ & $\begin{array}{l}\text { Facilidad del informante para } \\
\text { explicar su conocimiento }\end{array}$ \\
\hline
\end{tabular}

\section{Valoración de la parrilla}

Como se ha explicado arriba, la valoración de la parrilla es con enteros entre 1 y 5 , correspondientes a los polos bajo y alto, respectivamente, de los constructos o características considerados. Por ejemplo, un valor 1 en el par (Entrevista no estructurada/Experiencia en educción) indicará que es suficiente una baja experiencia en educción por parte del eductor para obtener efectividad de la técnica. Es decir, la técnica de Entrevista no Estructurada es adecuada incluso aplicada por eductores que presentan baja Experiencia en Educción. Por el contrario, un valor 5 indicará que la Entrevista no Estructurada requiere de una alta Experiencia en Educción por parte del eductor para que sea efectiva. Dicho de otra forma, para que la técnica Entrevista no Estructurada sea adecuada el eductor debe tener alta Experiencia en Educción.

Los baremos intermedios corresponden a valores medio-bajo (2), medio (3) y medio-alto (4). La estimación de estos valores no es absoluta, necesariamente, es decir, se deja a la consideración subjetiva de los sujetos la definición de estos rangos. Esto, debido a que es justamente la visión propia de cada sujeto de estudio la que se quiere capturar por medio de la técnica de emparrillado. Cabe notar que la parrilla no representa datos del sujeto que la está cumplimentando sino su opinión acerca de la adecuación de técnicas por cada constructo. Por ejemplo, el atributo experiencia no se refiere a la que posee el sujeto experimental, en este caso los ingenieros noveles o expertos, sino se refiere a si ellos consideran que un valor dado de experiencia del ingeniero de requisitos es necesaria para la aplicación de la técnica en cuestión

El diseño de la parrilla para la presente investigación se muestra en la Tabla 5.

Tabla 5. Parrilla resultante para estudio comparativo.

\begin{tabular}{|c|c|c|c|c|c|c|c|c|c|c|c|c|c|}
\hline & & E1 & $\mathbf{E 2}$ & $\mathbf{E 3}$ & $\mathbf{E 4}$ & $\mathbf{E 5}$ & E6 & E7 & E8 & E9 & E10 & E11 & E12 \\
\hline & & 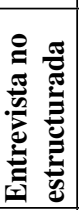 & 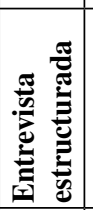 & 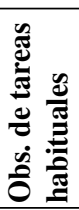 & 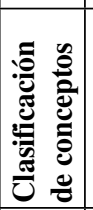 & 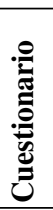 & 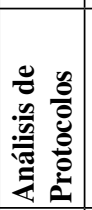 & 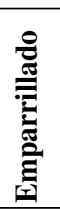 & 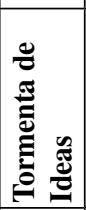 & 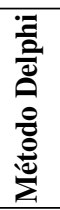 & 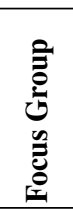 & 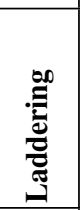 & 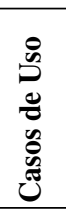 \\
\hline C1 & $\begin{array}{l}\text { Experiencia en educción } \\
\text { del eductor }\end{array}$ & & & & & & & & & & & & \\
\hline C2 & $\begin{array}{l}\text { Familiaridad con el } \\
\text { dominio del eductor }\end{array}$ & & & & & & & & & & & & \\
\hline C3 & $\begin{array}{l}\text { Disponibilidad de tiempo } \\
\text { de los informantes }\end{array}$ & & & & & & & & & & & & \\
\hline $\mathrm{C4}$ & Interés del informante & & & & & & & & & & & & \\
\hline C5 & \begin{tabular}{|l|} 
Nivel de pericia del \\
informante
\end{tabular} & & & & & & & & & & & & \\
\hline C6 & $\begin{array}{l}\text { Consenso entre } \\
\text { informantes }\end{array}$ & & & & & & & & & & & & \\
\hline C7 & $\begin{array}{l}\text { Capacidad de articulación } \\
\text { del informante }\end{array}$ & & & & & & & & & & & & \\
\hline
\end{tabular}




\section{EMPARRILLADOS NOVEL Y EXPERTO}

\section{Parrilla de los ingenieros noveles}

Los ingenieros de requisitos noveles fueron representados por un grupo de estudiantes de master en ingeniería de software. Todos los sujetos tenían similar experiencia en desarrollo y no tenían conocimiento de las técnicas de educción más allá de las entrevistas. Estos ingenieros informáticos y de profesiones afines recibieron una formación de 9 horas sobre el proceso de educción de requisitos software y sobre las técnicas de educción que pueden utilizarse en las sesiones; además de extensa documentación de apoyo.

Luego de esta instrucción se les pidió valorar una parrilla similar a la de la Tabla 5 previa información acerca de la finalidad del ejercicio. Las parrillas obtenidas fueron consensuadas en una sola que representa la visión del grupo. Esta parrilla se estableció por medio de la moda de los valores otorgados por los sujetos. Cuando hay más de un valor modal se considera la mediana de los valores moda.

La Tabla 6 muestra la parrilla resultante de los ingenieros noveles.

Tabla 6. Parrilla resultante de los noveles.

\begin{tabular}{|l|c|c|c|c|c|c|c|c|c|c|c|c|}
\hline & E1 & E2 & E3 & E4 & E5 & E6 & E7 & E8 & E9 & E10 & E11 & E12 \\
\hline C1 & 5 & 3 & 3 & 4 & 4 & 3 & 4 & 4 & 3 & 4 & 3 & 3 \\
\hline C2 & 2 & 4 & 1 & 4 & 4 & 4 & 4 & 2 & 4 & 3 & 4 & 3 \\
\hline C3 & 5 & 4 & 1 & 2 & 4 & 2 & 4 & 4 & 4 & 4 & 3 & 4 \\
\hline C4 & 4 & 4 & 2 & 3 & 4 & 4 & 3 & 5 & 4 & 4 & 4 & 3 \\
\hline C5 & 3 & 3 & 4 & 4 & 3 & 4 & 3 & 4 & 3 & 4 & 5 & 3 \\
\hline C6 & 3 & 1 & 3 & 4 & 1 & 3 & 1 & 5 & 4 & 5 & 4 & 2 \\
\hline C7 & 5 & 5 & 1 & 4 & 4 & 5 & 2 & 4 & 4 & 4 & 3 & 2 \\
\hline
\end{tabular}

\section{Parrilla de los expertos}

La visión experta fue representada por tres profesores encargados del módulo de requisitos del master en ingeniería de software. Estos sujetos de estudio tienen vasta experiencia tanto práctica como teórica en desarrollo de software y, en particular, en educción de requisitos. Ellos han impartido el módulo de requisitos del programa de máster alrededor de diez años y han participado en más de cinco proyectos como ingenieros de requisitos.
A estos expertos se les pidió completar la misma parrilla suscrita para los noveles y posteriormente fueron resumidas en una única parrilla con las modas de los valores otorgados. Igualmente que en el caso de la parrilla de noveles, si hay varias modas se considerará la mediana entre ellas. La Tabla 7 presenta esta parrilla representativa de la visión experta.

\section{DISCUSIÓN DE LOS RESULTADOS}

\section{Visión de los ingenieros noveles}

$\mathrm{Al}$ procesar la parrilla en la herramienta Webgrid 5 se obtiene la Figura 2, como salida del análisis de conglomerados.

Tabla 7. Parrilla resultante de los expertos.

\begin{tabular}{|c|c|c|c|c|c|c|c|c|c|c|c|c|}
\hline & E1 & E2 & E3 & E4 & E5 & E6 & E7 & E8 & E9 & E10 & E11 & E12 \\
\hline C1 & 5 & 3 & 2 & 4 & 1 & 5 & 2 & 4 & 4 & 5 & 3 & 4 \\
\hline C2 & 1 & 5 & 1 & 5 & 4 & 2 & 4 & 2 & 4 & 3 & 3 & 2 \\
\hline C3 & 4 & 4 & 1 & 4 & 1 & 5 & 3 & 5 & 1 & 5 & 4 & 5 \\
\hline C4 & 5 & 2 & 2 & 2 & 2 & 4 & 3 & 4 & 2 & 4 & 2 & 4 \\
\hline C5 & 2 & 2 & 1 & 1 & 1 & 4 & 2 & 2 & 2 & 2 & 1 & 3 \\
\hline C6 & 4 & 4 & 1 & 2 & 2 & 4 & 3 & 2 & 1 & 2 & 2 & 3 \\
\hline C7 & 5 & 3 & 1 & 1 & 1 & 5 & 2 & 3 & 1 & 3 & 1 & 4 \\
\hline
\end{tabular}

En la gráfica de las técnicas de educción se pueden distinguir algunos grupos con claridad. Por un lado, se puede observar que para los ingenieros noveles las técnicas tormenta de ideas y focus group parecen similares. Ellas presentan un alto porcentaje de equiparación $(92,9)$. El mismo porcentaje de similitud presenta la pareja entrevista estructurada y cuestionarios.

En este mismo conjunto aparecen relacionadas las técnicas emparrillado y casos de uso, y cuestionarios y emparrillado con una similitud del 89,3\%. Por otro lado, Las técnicas clasificación de conceptos y análisis de protocolos aparecen reunidas con un $85,7 \%$ de equiparación.

Respecto a los constructos o características, para los ingenieros noveles los atributos experiencia en educción del eductor, disponibilidad de tiempo del informante y nivel de pericia del informante parecen levemente similares, con un $83,3 \%$ de equiparación.

De igual forma, parecen levemente equiparados los atributos contextuales familiaridad con el dominio 
del eductor e interés del informante con un 81,2\% de similitud.

\section{Visión del experto}

Al procesar la parrilla en la herramienta Webgrid 5 se obtiene la Figura 3, como salida del análisis de conglomerados. Para los expertos, las técnicas de educción más similares en efectividad son tormenta de ideas y focus group. Estas técnicas presentan un $92,9 \%$ de equiparidad. La técnica tormenta de ideas también presenta una similitud, aunque menor $(89,3 \%)$, con casos de uso. A su vez, casos de uso parece similar a análisis de protocolos $(85,7 \%)$.

Por otro lado, es notoria la similitud entre las técnicas clasificación de conceptos y Laddering, que presentan una equiparación de $89,3 \%$. Finalmente, una similitud, aunque menor, del $82,1 \%$ fue encontrada entre las técnicas observación de tareas habituales, cuestionarios y método Delphi.

Con respecto a los constructos o características, los expertos perciben una leve similitud $(83,3 \%)$ entre familiaridad con el dominio del eductor e interés del informante.

También es detectada una cierta similitud entre los atributos Nivel de pericia del informante, capacidad de articulación del informante y consenso entre informantes $(81,2 \%)$.

\section{Discusión}

Al comparar las gráficas se puede observar que para los ingenieros noveles existe una visión más confusa, especialmente de las técnicas, que para los expertos. Las técnicas para los expertos conforman grupos más claros de distinción, en cambio los grupos de técnicas para los noveles presentan mayor similitud: casi todos los valores de equiparación de los grupos tienen valores sobre $85 \%$.

En la gráfica de las técnicas de educción de los expertos hay tres grupos claramente distinguibles. Sin embargo, no existe una explicación única sobre esta alineación diferenciada. Aunque puede caerse en la tentación de buscar como explicación el grado de estructuración de las técnicas debe notarse que éste no es un atributo de contexto, sino más bien una característica intrínseca de ellas. Si bien es cierto puede percibirse una correlación entre esta estructuración y las agrupaciones es probable que la configuración de técnicas cambie al agregar otros atributos contextuales al estudio. Recuérdese que solo se consideraron los atributos relacionados con los participantes en el proceso de educción.

En particular, en relación a las técnicas de educción, ambas visiones coinciden en destacar la relación entre las técnicas Tormenta de Ideas y Focus Group. Es decir, tanto para los ingenieros noveles como para los expertos estas técnicas parecen similares en efectividad.

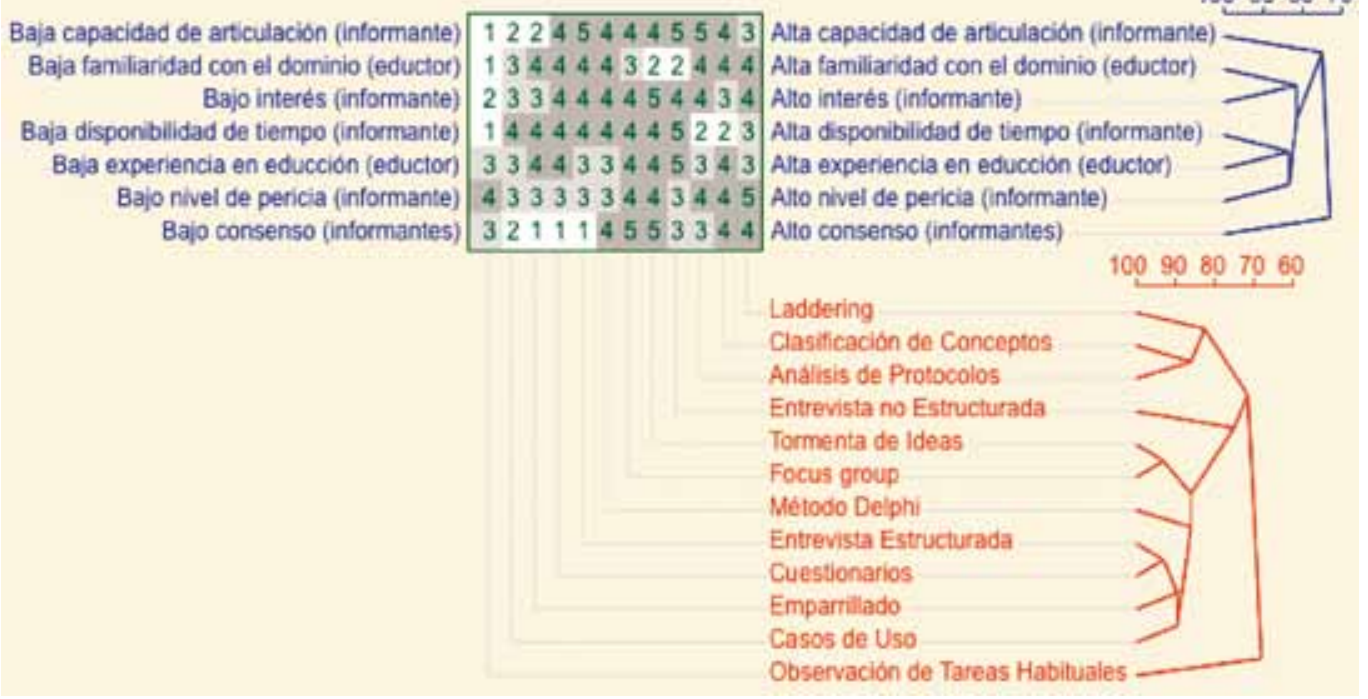

Figura 2. Análisis de conglomerados para los noveles. 


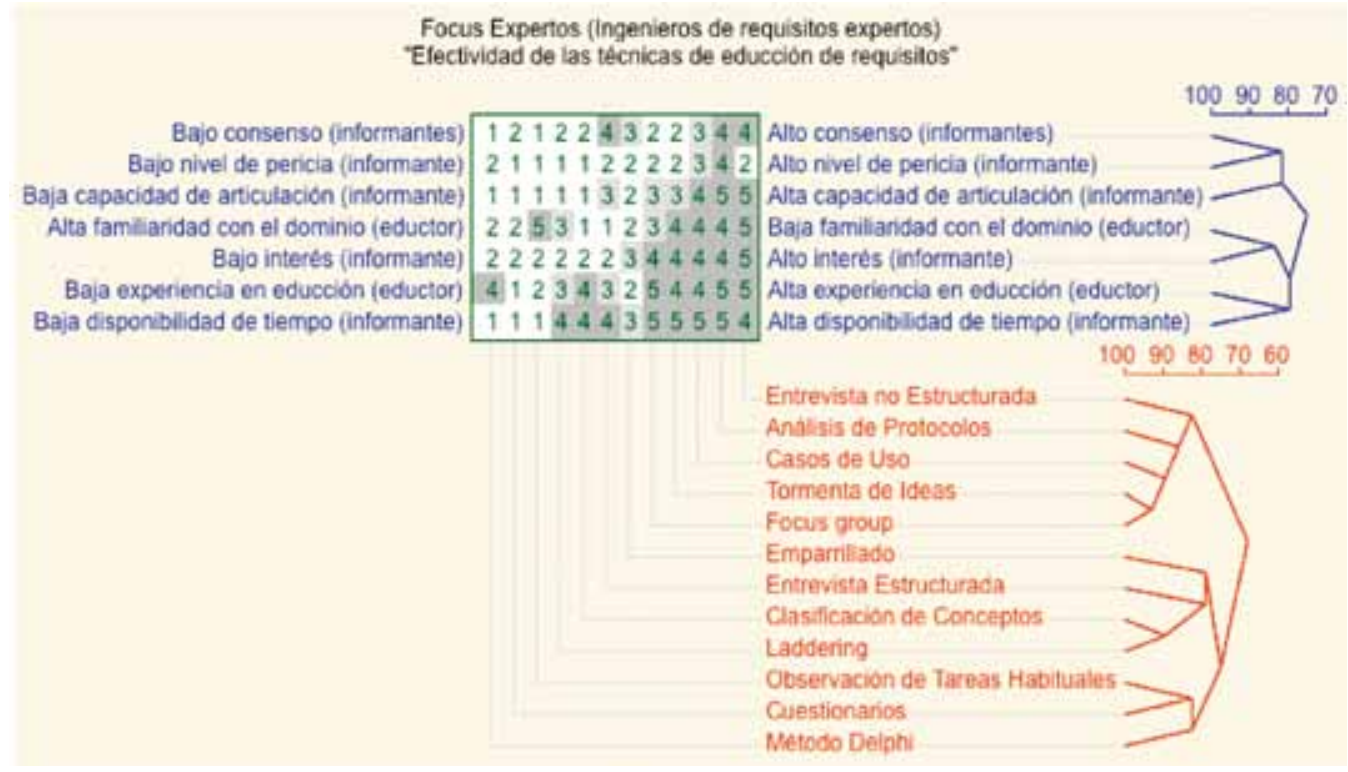

Figura 3. Análisis de conglomerados para los expertos.

El resto de la visión experta no es percibida por los noveles. Especialmente, la similitud entre clasificación de conceptos y Laddering, casos de uso y análisis de protocolos, y entre cuestionarios y método Delphi que, por naturaleza son claramente similares, no es compartida por la visión novel.

En relación a los atributos contextuales hay mayor coincidencia entre ambas visiones. Tanto para noveles como expertos, parecen similares la influencia de la experiencia en educción del eductor y la disponibilidad de tiempo del informante, y de familiaridad con el dominio del eductor y el interés del informante. Sin embargo, en este último caso, para los expertos la efectividad de las técnicas es similar cuando el interés del informante aumenta y la familiaridad del dominio baja. Esto significa que las técnicas consideradas son más adecuadas cuando hay menor conocimiento del dominio del problema por parte del eductor.

\section{Amenazas a la validez}

Este es un estudio exploratorio y por lo tanto tiene muchas amenazas a la validez de sus resultados.

En primer lugar, la técnica de emparrillado se ha simplificado proponiendo los constructos en vez de obtenerlos mediante el método original triadic. No obstante, para los fines del estudio esto no parece una amenaza relevante a la validez interna.
Aunque los resultados no pueden ser generalizados a la población de noveles y expertos, demuestran la viabilidad de la técnica como medio de comparación y evaluación indirecta. Ambas muestras son claramente de conveniencia pero teniendo características similares representan una baja amenaza a la validez externa.

La validez estadística es poco relevante ya que, debido a lo reducido de las muestras y a la baja potencia estadística, el trabajo ha preferido un estudio cualitativo que permite obtener tendencias empíricas.

Finalmente, la validez de constructo es la mayor amenaza al trabajo ya que los sujetos pueden no entender los constructos del emparrillado y evaluar erróneamente el concepto de efectividad de las técnicas. Se ha intentado entregar un entrenamiento previo a los sujetos sobre estos constructos para evitar confusiones.

\section{CONCLUSIONES Y TRABAJO FUTURO}

El estudio pretendía comparar las visiones de los ingenieros noveles y de expertos en requisitos software. Para ello se utilizó la técnica de emparrillado para reconocer cómo estos agentes perciben la efectividad de las técnicas de educción de requisitos. Aunque el estudio se centró en las técnicas de 
educción, también se obtuvieron percepciones acerca de las similitudes entre los atributos contextuales considerados.

Los resultados demostraron una sustancial diferencia entre ambas visiones respecto a la efectividad de las técnicas. Esto implica que una más amplia formación, y principalmente de tipo práctica, es necesaria para que los ingenieros noveles puedan decidir con mayor certeza sobre las técnicas más adecuadas a utilizar en las sesiones de educción de requisitos. Esto parece una perogrullada pero no existe evidencia alguna que apoye la mayoría de las creencias sobre las que basamos la construcción de sistemas software, de manera que confirmar empíricamente y cuantitativamente es mandato del método científico a considerar.

Una mayor coincidencia entre las visiones se detectó con respecto a los atributos del contexto que se consideraron en el estudio. Hay atributos que parecen influir similarmente en la efectividad de las técnicas de educción.

Las diferencias y similitudes arrojadas por el estudio se explican por la percepción de los sujetos sobre la influencia de los atributos en la efectividad de las técnicas. Sin embargo, el conocimiento descriptivo que tienen los ingenieros noveles sobre las técnicas puede llevarlos a confundir aspectos como su estructuración u origen con los resultados de su uso. Esa confusión se va aclarando con la experiencia práctica que permite encapsular las características intrínsecas de las técnicas y centrarse en la efectividad de su aplicación.

Los resultados de este estudio son un fundamento empírico para la preparación de estrategias en la formación práctica de las técnicas de educción. También es una ayuda para la preparación de otros estudios experimentales que permitan generar evidencias estadísticas acerca de la efectividad de las técnicas de educción.

En futuros trabajos se pretende extender este estudio exploratorio aumentando la cantidad de técnicas y atributos contextuales considerados. De esta forma, se tendría las visiones definitivas y en base a ello, la formulación de estrategias de entrenamiento e investigación empírica.

\section{AGRADECIMIENTOS}

Sinceros agradecimientos para los estudiantes del máster en ingeniería de software de la Universidad Politécnica de Madrid que participaron desinteresadamente en este estudio. También agradezco la colaboración de los profesores del módulo de requisitos que participaron como expertos.

\section{REFERENCIAS}

[1] AA. Abran and J.W. Moore. "SWEBOK: Guide to the Software Engineering Body of Knowledge (2004 edition)”. 2004. URL: http://www.computer.org/portal/web/swebok. Date of visit: May 30, 2005.

[2] H.F. Hofmann and F. Lehner. "Requirements engineering as a success factor in software projects". IEEE Software. Vol. 18, Issue 4, pp. 58-66. July-Aug., 2001.

[3] P. Chatzoglou and L. Macaulay. "Requirements capture and IS methodologies". Information Systems Journal. Vol. 6, Issue 3, pp. 209-225. July, 1996.

[4] J. Goguen and C. Linde. "Techniques for requirements elicitation". International Symposium on Requirements Engineering. Los Alamitos, California: IEEE Computer Society Press, pp. 152-164. January, 1993.

[5] G. Playle and C. Schroeder. "Software requirements elicitation: Problems, tools, and techniques". Crosstalk: The Journal of Defense Software Engineering. Vol. 9, Issue 12, pp. 19-24. December, 1996.

[6] H. Saiedian and R. Dale. "Requirements engineering: making the connection between the software developer and customer". Information and Software Technology. Vol. 42, Issue 6, pp. 419-28. April, 2000.

[7] B. Nuseibeh and S.M. Easterbrook. "Requirements engineering: A roadmap". In A.C.W. Finkelstein, ed., The Future of Software Engineering (Companion volume to the proceedings of the 22nd International Conference on Software Engineering, ICSE'00). IEEE Computer Society Press. 2000.

[8] A. Davis and P. Hsia. "Giving voice to requirements engineering: Guest editors' introduction". IEEE Software. Vol. 11, Issue 2, pp. 12-16. March, 1994. 
[9] G.A. Kelly. "The Psychology of Personal Constructs". New York: Norton. 1955.

[10] P. McGeorge and G. Rugg. "The uses of 'contrived' knowledge elicitation techniques". Expert-Systems. Vol. 9, Issue 3, pp. 149-154. August, 1992.

[11] B. Gonzalez-Baixauli, M. Laguna and J.C.S. do Prado Leite. "Applying Personal Construct Theory to Requirements Elicitation". IEEE Latin America Transactions. Vol. 3, Issue 1. March, 2005.

[12] "Webgrid 5". Fecha de actualización: 23 de marzo de 2010. URL: http://gigi.cpsc. ucalgary.ca:2000/. Fecha de consulta: 30 de junio de 2010.

[13] O. Dieste, A. Davis, A. Hickey, N. Juristo and A.M Moreno. "Effectiveness of Requirements Elicitation Techniques: Empirical Results Derived from a Systematic Review". Proc. of 14th IEEE International Conference on Requirements Engineering. September 11-15, 2006.

[14] D. Carrizo, O. Dieste and N. Juristo. "Study of elicitation techniques adequacy”. Proceedings XI Workshop on Requirements Engineering, WER 2008. Barcelona, Spain. September, 2008.

[15] S. Young, M. Edwards, S. McDonald and J. Barrie. "Personality characteristics in an XP team: a repertory grid study". HSSE '05: Proceedings of the workshop on Human and social factors of software engineering. Publisher: ACM. July, 2005.

[16] V.A. Khamisani, M.S. Siddiqui and M.Y. Bawany. "Analyzing Soft Skills of Software Engineers using Repertory Grid". IEEE Multitopic Conference, pp. 259-264. 2006.

[17] L.A. Macaulay. "Requirements Engineering". Berlin: Springer Verlag Series on Applied Computing. 1996.

[18] M. Christel and K. Kang. "Issues in Requirements Elicitation". SEI Technical Report No: SEI-92-TR-012, Carnegie Mellon Software Engineering Institute. September, 1992.

[19] J. Preece, Y. Rogers, H. Sharp, D. Benyon, S. Holland and T. Carey. "Human-Computer Interaction”. Reading MA, Addison-Wesley. 1994.

[20] D. Maurer and T. Warfel. "Card Sorting: A Definitive Guide”. Boxes and Arrows. 2004.
[21] F.K. Shuttleworth. "A study of Questionnaire technique". Journal of Educational Psychology. Vol. 22, pp. 652-658. December, 1981.

[22] K.A. Ericsson and H.A. Simon. "Protocol Analysis: Verbal Reports as Data". Cambridge, MA: MIT Press. 1984.

[23] M. Easterby-Smith. "The design, analysis and interpretation of repertory grid". In M.L.G. Shaw, Eds. Recent Advances in Personal Construct Psychology. Academic Press. London. 1983.

[24] A.F. Osborn. "Applied Imagination". New York: Charles Scribner's Sons. 1953.

[25] H.A. Linstone and M. Turoff. "The Delphi Method". Addison-Wesley Publishing Co. Inc. Londres. 1977.

[26] D.L. Morgan. "Successful Focus Groups: Advancing the State of the Art". Newbury Park, Calif.: Sage. 1993.

[27] G. Rugg and P. McGeorge. "Laddering knowledge elicitation". Expert-Systems. Vol. 12, Issue 4, pp. 339-346. November, 1995.

[28] I. Jacobson. "Object Oriented Development in an Industrial Environment". OOPSLA. 1987.

[29] J. Kim and J. Courtney. "A survey of knowledge acquisition techniques and their relevance to managerial problem domains". Decision Support Systems. Vol. 4, pp. 269284. 1988.

[30] J.S. Dhaliwal and I. Benbazat. "A framework for the comparative evaluation of knowledge acquisition tools and techniques". Knowledge Acquisition. Vol. 2, Issue 2, pp.145-166. June, 1990.

[31] T.A. Byrd, K.L. Cossick and R.W. Zmud. "A synthesis of research on requirements analysis and knowledge acquisition techniques". MIS Quarterly. Vol. 16, pp. 117-138. 1992.

[32] N. Maiden and G. Rugg. "ACRE: selecting methods for requirements acquisition". Software Engineering Journal. Vol. 11, Issue 3, pp. 183-192. May, 1996.

[33] S. Robertson and J. Robertson. "Mastering the requirements process". Addison-Wesley. 1999.

[34] A. Davis and A. Hickey. "A tale of two ontologies: The basis for systems analysis technique selection". Proc. 9th Annual 
American Conference on Information System. 2003.

[35] S. Lauesen. "Software requirements: Styles and techniques". Addison-Wesley. 2002.

[36] D. Zowghi and C. Coulin. "Requirements Elicitation: A Survey of Techniques, Approaches, and Tools". In A. Aurum and C. Wohlin (eds.). Book chapter in Engineering and Managing software requirements. SpringerVerlag, pp. 19-46. Springer, New York. 2005.
[37] Li Jiang, A. Eberlein and B.H. Far. "A case study validation of a knowledge-based approach for the selection of requirements engineering techniques". Requirements Engineering. Vol. 13, Issue 2, pp. 117-146. 2008.

[38] E. Kheirkhah and A. Deraman. "Important factors in selecting Requirements Engineering Techniques". International Symposium on Information Technology. 2008. 